\title{
Retraction Note to: Numerical simulation of inertial alfven waves to study localized structures and spectral index in auroral region
}

\author{
BHEEM SINGH JATAV \\ Department of Physics, Sikkim University, Gangtok, Sikkim 737101, India. \\ E-mail: bheemsinghj@gmail.com \\ published online 17 June 2020
}

Retraction to: J. Astrophys. Astr. (2018) 39:37

https://doi.org/10.1007/s12036-018-9531-8

From a report based on an independent investigation by a senior fellow of the Indian Academy of Sciences, we find that there is evidence of academic misconduct in the paper titled, "Numerical simulation of inertial alfven waves to study localized structures and spectral index in auroral region" by Bheem Singh Jatav (https:// doi.org/10.1007/s12036-018-9531-8; volume 39 article ID 37) published in Journal of Astrophysics and Astronomy. Therefore, the Indian Academy of Sciences has decided to withdraw the publication, on the grounds of academic misconduct by the author. The author has been notified and has agreed to the retraction. 